\title{
MicroRNA-24-3p Attenuates Myocardial Ischemia/Reperfusion Injury by Suppressing RIPK1 Expression in Mice
}

\author{
Hong Tan ${ }^{\mathrm{a}}$ Jie Q ${ }^{\mathrm{b}} \quad$ Bo-Yuan Fan ${ }^{\mathrm{c}}$ Jian Zhang ${ }^{\mathrm{b}}$ Fei-Fei Su ${ }^{\mathrm{d}}$ Hong-Tao Wang ${ }^{\mathrm{c}}$ \\ aCollege of Life Sciences, Northwest University, Xi'an, bSchool of Basic Medical Sciences, Xi'an Jiaotong \\ University, Xi'an, 'Department of Cardiology, the Second Affiliated Hospital, Xi'an Jiaotong University, \\ Xi'an, ${ }^{d}$ Department of Cardiology, Tangdu Hospital, Fourth Military Medical University, Xi'an, China
}

\section{Key Words}

miR-24-3p • RIPK1 • Myocardial ischemia reperfusion injury

\begin{abstract}
Background/Aims: This study was developed to investigate a potential therapeutic method for myocardial ischemia/reperfusion injury involving the promotion of miR-24-3p expression. Methods: Microarray analysis was used to screen differentially expressed genes in a myocardial ischemia/reperfusion (I/R) injury mouse model. Gene set enrichment analysis was utilized to determine vital signaling pathways. Targeting verification was conducted with a luciferase reporter assay. Myocardial I/R injury was developed in mice, and the expression levels of RIPK1 and miR-24-3p were investigated by qRT-PCR and Western blot. Hemodynamic parameters and the activity of serum myocardial enzymes were measured to evaluate cardiac function. Infarct area was observed through HE and TTC staining. Myocardial cell apoptosis was examined by TUNEL staining and caspase-3 activity analysis. Results: RIPK 1 was an upregulated mRNA found by microarray analysis and a verified target of the downregulated miRNA miR-24-3p. The upregulation of RIPK1 (1.8-fold) and the downregulation of miR-24-3p (0.3-fold) were confirmed in I/R mice. RIPK1 led to impaired cardiac function indexes, increased infarct area and cell apoptosis, while miR-24-3p could reverse the injury by regulating RIPK1. The TNF signaling pathway was proven to be involved in myocardial I/R injury through the detection of the dysregulation of related proteins. Conclusion: In conclusion, RIPK1 was upregulated and miR-24-3p was downregulated in a myocardial I/R injury mouse model. RIPK 1 could aggravate myocardial I/R injury via the TNF signaling pathway, while miR-24-3p could suppress RIPK 1 and therefore exert cardioprotective effects in myocardial I/R injury.
\end{abstract}




\section{Cellular Physiology Cell Physiol Biochem 2018;51:46-62

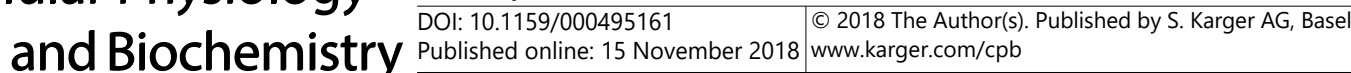 \\ Tan et al.: Role of miR-24-3p in Myocardial Ischemia/Reperfusion Injury}

\section{Introduction}

Myocardial ischemia and reperfusion are pathological conditions characterized by a lack of blood supply to the heart and subsequent restoration of perfusion together with reoxygenation [1]. It is commonly recognized that the reestablishment of blood flow in ischemic myocardial tissue has become one of the most viable therapeutic approaches for ischemic heart disease [2]. Perhaps surprisingly, the recovery of blood supply and reoxygenation are always related to the exacerbation of tissue injury and inflammatory responses, which are together called reperfusion injury [3]. The efficacy of the therapies for heart disease might be affected by subsequent ischemia/reperfusion (I/R) injury, which could result in serious consequences, such as apoptosis and necrosis of myocytes or even cardiac arrest [1]. There have been numerous studies, and clinical evidence suggests that I/Rinduced cardiac injury is associated with gene regulation [4,5]. Although the mechanisms of myocardial I/R injury are well understood and have been demonstrated in several studies, the application of those findings to clinical research has been insufficient.

Microribonucleic acids (miRNAs) are small and highly conserved noncoding RNAs with approximately 22 nucleotides. MiRNAs can modulate gene expression at the posttranscriptional level by promoting the degradation or inhibiting the translation of messenger RNAs (mRNAs) by targeting their 3' untranslated regions (UTRs) [6]. It is widely accepted that a single miRNA can influence multiple gene transcripts to regulate the complex process of gene expression, ultimately affecting the physiology of a cell [5]. Hence, miRNAs have been regarded as main regulators in almost all cellular processes, including differentiation, proliferation, necrosis and apoptosis [7, 8]. Zhu WS et al. investigated the role of miR-1 in I/R-induced injury and found that the suppression of miR-1 might be protective against myocardial I/R injury [9]. Zhu J et al. found miR-499 protects the rat heart against I/R injury by inhibiting apoptosis through PDCD4 [10]. Wang XH et al. reported the function of miR-494 in cardiac pathophysiological processes and revealed the protective role of miR494 in I/R therapy [8]. Moreover, Lorenzen JM et al. investigated the expression of miR-24 in I/R injury in kidneys and in a mouse model and concluded that miR-24 regulation could be regarded as a diagnostic index for acute kidney injury [11]. Accordingly, miRNAs may be able to be treated as novel biomarkers and might be promising as diagnostic tools for cardiovascular diseases.

In this study, we also observed that Receptor-interacting serine/threonine-protein kinase 1 (RIPK1) was markedly upregulated in a mouse model of I/R injury. It has been reported that cell survival or death can be regulated by several RIPK1-mediated pathways, such as the tumor necrosis factor (TNF) signaling pathway, which ultimately results in NF- $\mathrm{KB}$ expression and participation in the deoxyribonucleic acid (DNA) transcription process [12]. In addition to RIPK1, Ripk3 also participates in the regulation of necrotic death in particular cell types, and both RIPK1 and Ripk3 have been demonstrated to be activated in ischemic heart disease [13]. Oerlemans MI et al. were the first to demonstrate that the phosphorylation of RIPK1 is increased in the heart after ischemia/reperfusion, and the suppression of RIPK1dependent necrosis can inhibit adverse cardiac remodeling after I/R injury [14]. However, the molecular mechanism of RIPK1 in necrotic tissues and the targeting miRNAs are unclear and have yet to be elucidated.

In the present study, we reanalyzed pre-existing microarrays and established an induced I/R male mouse model to determine the molecules and biological signaling pathways that participate in the regulation of myocardial ischemia/reperfusion. The roles of RIPK1 and miR-24-3p were demonstrated by overexpression of these two biological macromolecules. Cardiac function, infarct area and myocardial cell apoptosis were observed to evaluate the extent of myocardial I/R injury. Our study investigated the possibility that RIPK1 and miR24-3p play important roles in providing cardioprotective effects against myocardial I/R injury. 


\section{Cellular Physiology Cell Physiol Biochem 2018:51:46-62

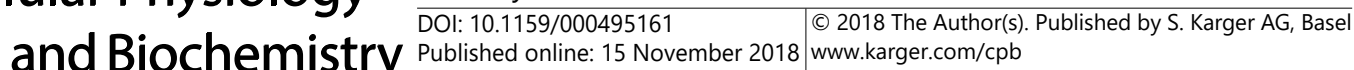

\section{Materials and Methods}

\section{Mouse model preparation}

In the present study, 102 male C57BL/6J mice at the age of 10-12 weeks were purchased from Charles River (Beijing, China). All experiments were performed under the protocol of the Use of Laboratory Animals from the National Institutes of Health Guidelines. Mice were fed a mouse diet and given tap water in a temperature-controlled room (12/12 h light/dark cycle). Twelve mice died of myocardial ischemia or adenovirus transfection. The other 90 mice were separated into five large groups (each group contained 18 mice), including (1) a sham group, (2) an I/R group, (3) an I/R + miR-24-3p mimics group, (4) an I/R + ad-RIPK1 group and (5) an I/R + miR-24-3p mimics + ad-RIPK1 group. The mice were examined by echocardiography and serum myocardial enzyme activity analysis after I/R or sham surgery, and then they were sacrificed. The myocardial tissues were collected and used for HE staining, TTC staining, transferasemediated dUTP nick end labeling (TUNEL) staining, caspase-3 activity analysis and protein expression level detection.

\section{Animal model construction of myocardial ischemia/reperfusion injury}

Male C57BL/6J male mice were screened for those of the same body weight and were then anesthetized with $100 \mathrm{mg} / \mathrm{kg}$ ketamine and $10 \mathrm{mg} / \mathrm{kg}$ xylazine before endotracheal intubation. Then, the left thoracic cavities of the mice were opened, and 8-0 Prolene sutures were used to stitch the left anterior descending (LAD) coronary arteries to produce occlusions. After the LAD coronary arteries were ligated, the ST segments of the mice exhibited persistent elevation. After 30 minutes of ischemia induction, the ligature was released, and the mice were reperfused for 60 minutes. Finally, the chests were stitched, and the endotracheal tubes were removed so that the mice could resume spontaneous respiration. In addition, the sham-operated group of mice experienced the same experimental procedure except the ligation of the left anterior descending coronary arteries. After the experiment, the mice were anesthetized with isoflurane and then sacrificed. Blood and myocardial tissues from the infarct zone were collected for quantitative and qualitative analyses and morphological observations.

\section{Microarray analysis}

The miRNA and mRNA microarray expression data have been deposited in the Gene Expression Omnibus of the National Center of Biotechnology Information (NCBI) with accession numbers GSE45818 and GSE50886. We reanalyzed the microarrays GSE45818 and GSE50886 through the GPL11533 and GPL6246 platforms, respectively. For GSE45818, we used three untreated heart control groups and three ischemic heart groups for mRNA microarray analysis. For GSE50886, we selected two control groups and three ischemic heart groups for miRNA microarray analysis. Differentially expressed miRNAs and mRNAs were screened using Student's $t$-test $(P$-value $<0.05)$ accompanied by $\mid \log 2$ (fold change) $\mid>1$. The results are shown on a heatmap and a volcano plot.

\section{Vector construction for adenovirus transfection}

For the delivery of miR-24-3p, male C57BL/6 mice received intravenous injections of miR-24-3p for three consecutive days. For the delivery of adenovirus, anesthetized mice were treated with an animal ventilator. Then, the chests of the mice were opened, and $2 \times 10^{7} \mathrm{pfu} / \mathrm{mL}$ ad-RIPK1 adenovirus was injected from the apex of the left ventricle into the aortic root. The pulmonary arteries were clamped, and the clamp was held for 20 seconds while the heart pumped in a closed system. After the air and blood were removed, the chests were stitched, and the mice were sent to cages for recovery. Five days after adenovirus injection, the mice underwent I/R surgery.

\section{Quantitative real-time polymerase chain reaction ( $Q R T-P C R$ )}

Total RNA was isolated with TRIzol reagent (Thermo Fisher Scientific, MA, USA) and reversetranscribed into complementary DNA (cDNA) before qRT-PCR analysis. U6 and reduced glyceraldehydephosphate dehydrogenase (GAPDH) expression served as the controls. The expression of miR-24-3p and RIPK1 was quantified using the $2^{-\Delta \Delta c t}$ method. The primers we used are listed in Table 1.

\section{KARGER}




\section{Cellular Physiology Cell Physiol Biochem 2018:51:46-62 and Biochemistry \begin{tabular}{l|l} 
DOI: 10.1159/000495161 2018 The Author(s). Published by S. Karger AG, Basel \\
Published
\end{tabular}

Western blot assay

Total protein was lysed using RIPA lysate. The protein concentration was measured using a Pierce BCA Protein Assay Kit (Pierce, Rockford, IL, USA). The proteins were transferred onto polyvinylidene fluoride (PVDF) membranes for SDSPAGE detection (concentration of $12 \%$ ). Afterwards, the membranes were blocked in 5\% nonfat milk at room temperature for $1 \mathrm{~h}$. Then, the membranes were incubated with primary antibodies against Fas (ab82419, 1:1000 dilution, Abcam), anti-TNF alpha (ab6671, 1:1000 dilution, Abcam) and anti-RIP (ab106393, 1:1000 dilution, Abcam) overnight at $4^{\circ} \mathrm{C}$. The membranes were washed three times using TBST and were then incubated with goat anti-rabbit secondary antibody (ab205718, 1/10000, Abcam) for $1.5 \mathrm{~h}$ at room temperature. The membranes were finally washed with TBST in triplicate and analyzed using an enhanced chemiluminescence (ECL) detection system (Thermo Fisher Scientific, USA).

\section{Luciferase reporter assay}

HEK293T cells at a density of $5 \times 10^{4}$ cells per well were seeded into a 24-well plate. The 3'UTR fragments of RIPK1 with both wild-type (wt) and mutant (mut) binding sites for miR-24-3p, with Sfi I cleavage sites on each side, were synthesized and subcloned into pGL4.10 luciferase reporter vectors (Promega). After incubating overnight, the cells were transfected with the constructed vector (10 ng) along with negative control mimics (50 ng) or miR-24-3p mimics (50 ng) using Lipofectamine 2000 (Thermo Fisher) according to the protocols of the manufacturer. The transfected cells were collected $48 \mathrm{~h}$ later. A dual luciferase reporter assay kit (Promega) was utilized for luciferase activity analysis, and the luciferase activity was detected via a SpectraMax L (Molecular Devices, CA, USA).

\section{Color Doppler Echocardiography}

Cardiac blood flow parameters including left ventricular end-diastolic dimension (LVEDD), left ventricular end systolic diameter (LVESD), left ventricular ejection fraction (LVEF), left ventricular fraction shortening (LVFS), left ventricular systolic pressure (LVSP), left ventricular end-diastolic pressure (LVEDP) and $\pm \mathrm{dp} / \mathrm{dt}_{\text {max }}$, were detected by ultrasound Doppler examination.

\section{Hematoxylin-eosin (HE) staining}

The previously collected myocardial tissues were fixed with $4 \%$ paraformaldehyde for $24 \mathrm{~h}$ according to standard procedures. Five millimeter thick sections were embedded in paraffin. Next, HE staining was utilized to measure the alterations in morphology in the myocardial tissues. The staining was observed under an optical microscope to visualize the degree of heart damage, and different fields were subsequently photographed.

\section{Determination of myocardial infarct size}

The myocardial infarct size was determined by staining with 2, 3,5-triphenyl tetrazolium chloride (TTC, TGC, Shanghai, China). Briefly, $3 \mathrm{~h}$ after reperfusion, the heart was quickly excised. The heart was then stored at $-80^{\circ} \mathrm{C}$ and sliced into small sections ( $1 \mathrm{~mm}$ thick). The slices were incubated with $1 \%$ TTC for 15 minutes at $37^{\circ} \mathrm{C}$. The infarct area (Inf, pale white) and the area at risk (AAR, brick red) were measured using Image-Pro Plus 6.0 software. The ratios of the Inf to the AAR and the AAR to the left ventricular are (LV) were calculated. 


\section{Cellular Physiology Cell Physiol Biochem 2018;51:46-62 and Biochemistry \begin{tabular}{l|l} 
DOI: 10.1159/000495161 & $\begin{array}{l}\text { (c) } 2018 \text { The Author(s). Published by S. Karger AG, Basel } \\
\text { www.karger.com/cpb }\end{array}$
\end{tabular} \\ Tan et al.: Role of miR-24-3p in Myocardial Ischemia/Reperfusion Injury}

Transferase-mediated dUTP nick end labeling (TUNEL) staining

A TUNEL assay was performed to evaluate cell apoptosis in myocardial tissues using a TUNEL method with a detection kit (Roche, Mannheim, Germany) according to the manufacturer's protocol. The nuclei that stained brown were considered TUNEL-positive cells. Five visual fields in each slice were randomly chosen, and the average number of apoptotic cells per 200 cells was determined. The apoptosis index (AI) was determined as follows: $\mathrm{AI}=$ (the number of positive cells/the total number of counted cells) $\times 100 \%$.

\section{Caspase-3 activity test}

To detect the induced apoptotic pathway, a caspase-3 activity assay kit (BestBio, Shanghai, China) was used according to the manufacturer's instructions. Myocardial tissues were treated with lysis buffer and homogenized with a tissue grinder. Subsequently, $90 \mu \mathrm{L}$ of reactionbuffer was added to each lysate extract. Finally, $10 \mu \mathrm{L}$ of Ac-DEVD-pNA (caspase-3 substrate) was added, and the absorbance of the samples was read at $405 \mathrm{~nm}$.

\section{Statistical analysis}

All the data in this study are expressed as the means \pm S.E.M. of at least three independent experiments. One-way analysis of variance was used for multiple comparisons. $P<0.05$ was considered significant.

\section{Results}

\section{Microarray analysis}

We set $\mid \log 2$ (fold change) $\mid>1$ and $P<0.05$ as the screening criteria to select the differentially expressed mRNAs. The 16 most upregulated mRNAs and the 4 most downregulated mRNAs were selected to draw the heatmap (Fig. 1A). We found that RIPK1 was one of the 16 upregulated mRNAs. The miRNA microarray analysis screened the 10 most upregulated and the 10 most downregulated miRNAs (Fig. 1B). miR-24-3p was found to be significantly downregulated in the ischemia/reperfusion mouse model, in contrast to the expression of RIPK1.

Gene ontology (GO) term enrichment analysis for differentially expressed genes

GO enrichment analysis was applied based on several biological functions to the differentially expressed genes identified in the microarray (Fig. 1C). We found many enriched biological processes, including vasculature development and transcription factor binding, that may play important roles in myocardial ischemia/reperfusion injury (Fig. 2A and Fig. 2B).

\section{KEGG pathway enrichment analysis}

KEGG pathway analysis was performed to investigate the biological signaling pathways involved in ischemia/reperfusion injury (Fig. 1D). According to the enrichment scores, the top 10 activated pathways in normal mice or I/R mice were identified. On the basis of the results in Fig. 3A and Fig. 3B, we found that the TNF signaling pathway was highly activated in the I/R mouse model, indicating a significant role for the TNF signaling pathway in myocardial ischemia/reperfusion injury. Additionally, the highly upregulated mRNA RIPK1, which was analyzed previously in the microarray, has been suggested as a component of the TNF signaling pathway, reassuring us of the involvement of RIPK1 in myocardial ischemia/ reperfusion injury.

\section{Validation of the targeting relationship between miR-24-3p and RIPK1}

In the analysis of hierarchical clustering (Fig. 4A), mRNAs involved in the TNF signaling pathway were confirmed to be differentially expressed in normal and I/R mice. It was notable that all the DEGs participating in the TNF signaling pathway were upregulated in the I/R model. The TargetScan database (Fig. 4B) predicted that miR-24-3p could bind to the 3'UTR of wild-type RIPK1, which suggested that miR-24-3p could serve as a suppressor of RIPK1. 


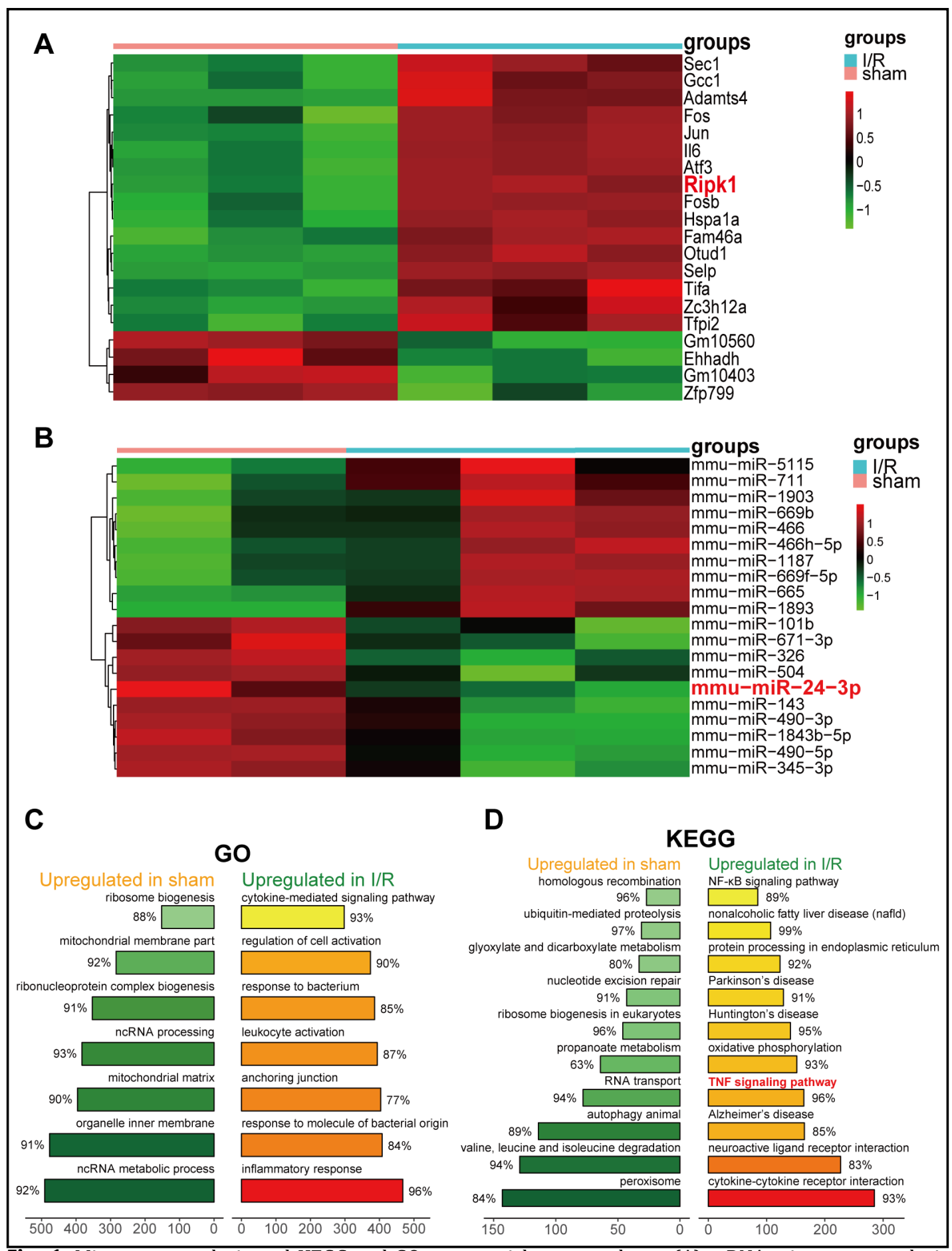

Fig. 1. Microarray analysis and KEGG and GO term enrichment analyses. (A) mRNA microarray analysis of ischemia/reperfusion $(\mathrm{P}<0.5$, fold change $>2) ; 16$ of the most upregulated mRNAs are presented using a heatmap. (B) mRNA microarray analysis of ischemia/reperfusion $(\mathrm{P}<0.5$, fold change $>2) ; 10$ of the most downregulated miRNAs are presented using a heatmap. (C) GSEA of GO (Gene Ontology) annotations. (D) KEGG analysis showed that the TNF pathway was highly expressed in the I/R mouse model. 


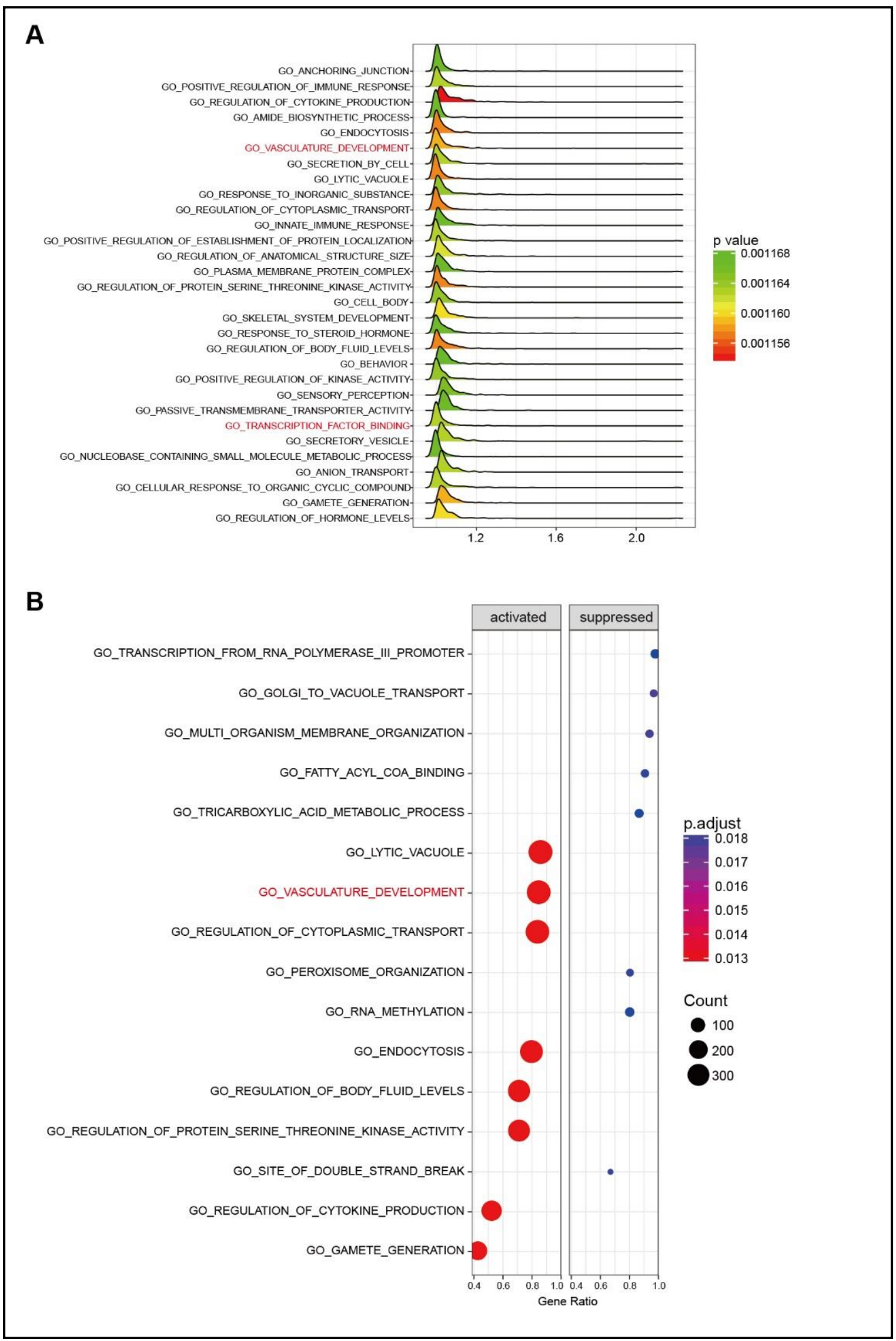

Fig. 2. GSEA of gene ontology. (A and B) Joy and dot plots of GO annotations illustrating the distributions of several cell functions. 
A

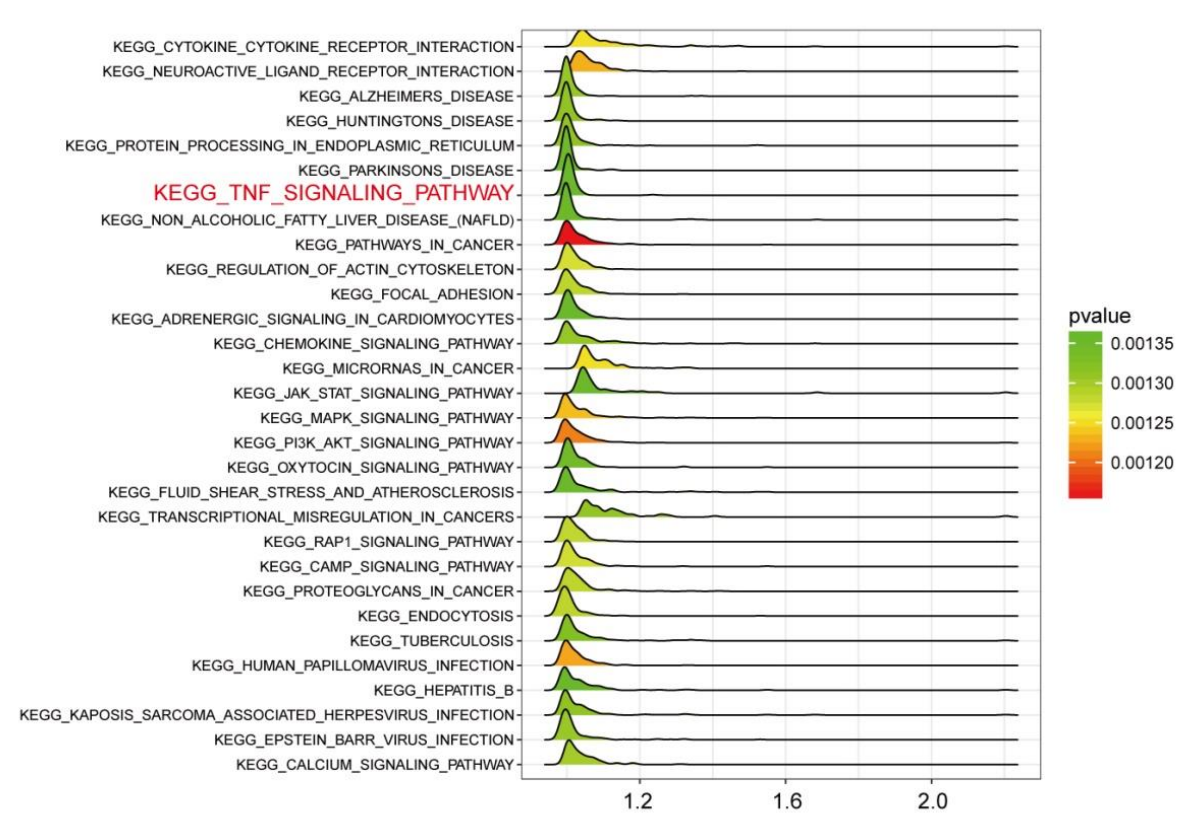

B

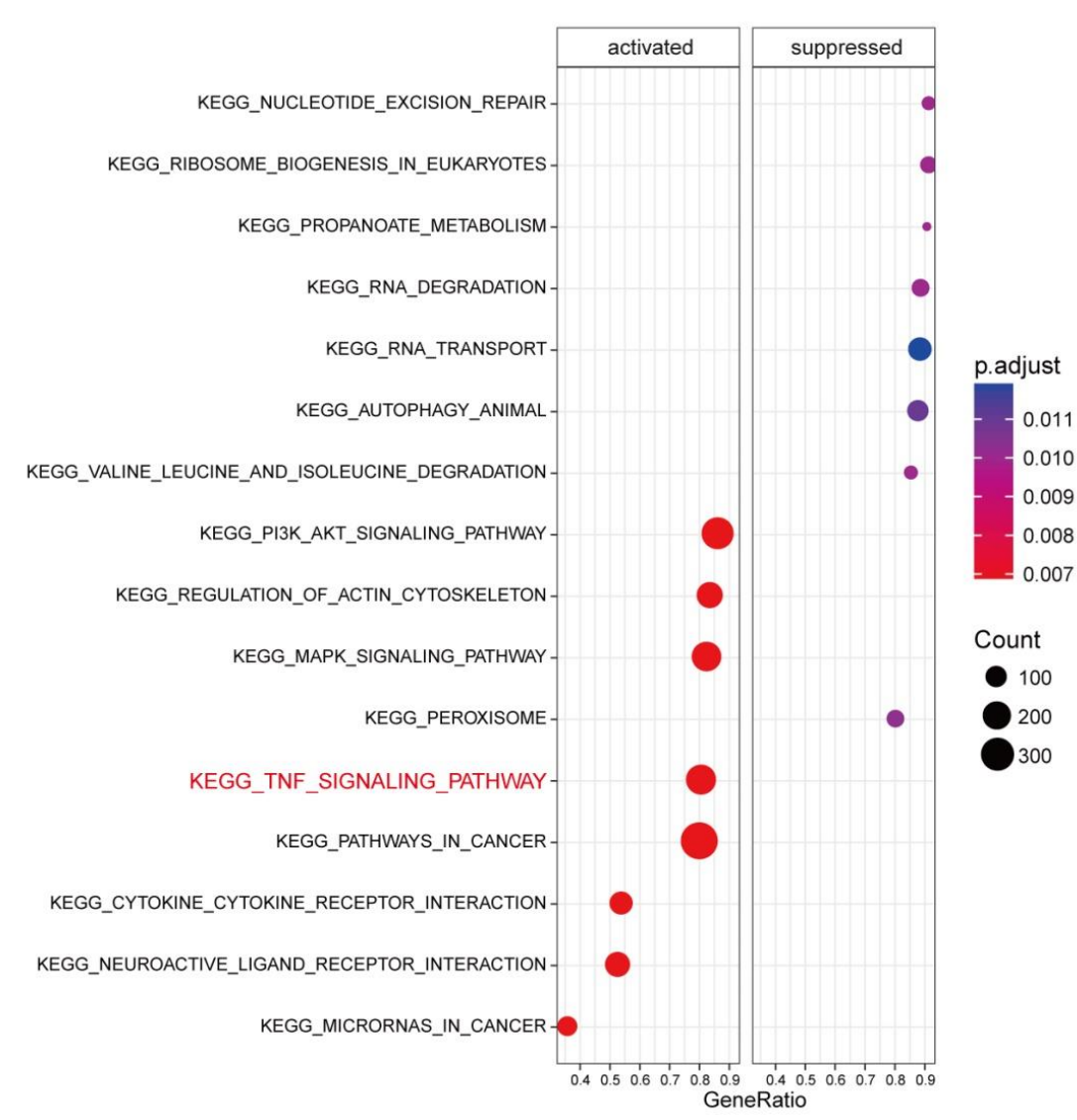

Fig. 3. GSEA of the KEGG pathway. (A and B) Joy and dot plots of KEGG annotations illustrating the distributions of several KEGG pathway gene sets among all the differentially expressed genes. 


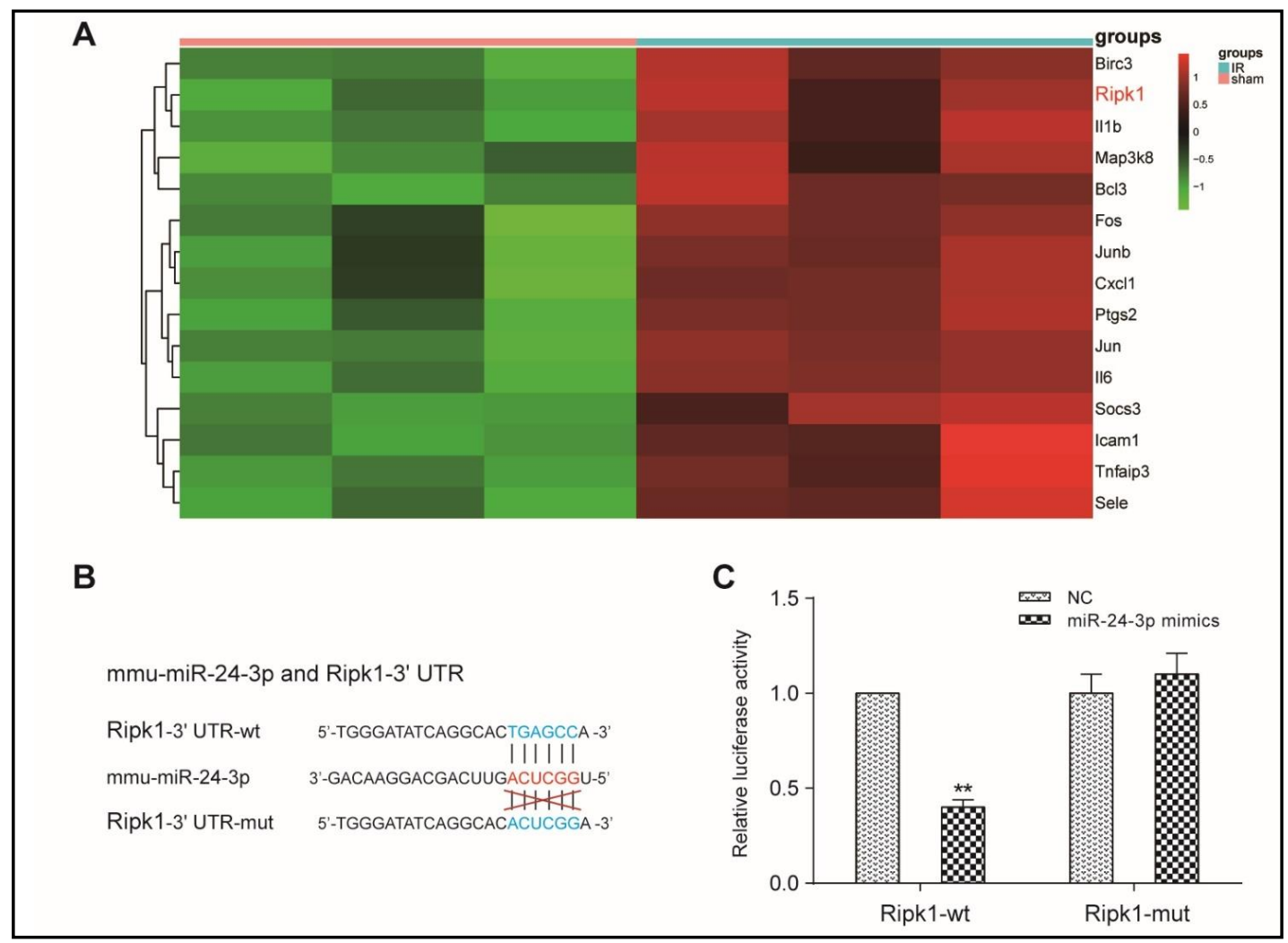

Fig. 4. Ripk1 was the target of miR-24-3p. (A) Hierarchical clustering analysis in the KEGG-TNF pathway showed that Ripk1 was highly expressed in the TNF pathway. (B) The TargetScan database predicted the binding site on Ripk1 that miR-24-3p targeted. (C) A dual luciferase reporter assay confirmed that transfection of miR-24-3p could decrease the luciferase activity of Ripk1-wt $(\mathrm{P}<0.01)$. (**indicates $\mathrm{P}<0.01$ compared with the NC group).

Subsequently, the targeting relationship between miR-24-3p and RIPK1 was confirmed by a dual luciferase reporter assay (Fig. 4C). The luciferase activity of the cells transfected with miR-24-3p in the RIPK1-wt group markedly decreased compared to that of the transfected cells in the RIPK1-mut group $(P<0.01)$.

The effects of RIPK1 and miR-24-3p on the expression levels of serum myocardial enzymes

Fig. 5A and 5B demonstrate the relative RNA expression levels of miR-24-3p and RIPK1. It was confirmed that in the I/R mouse model, miR-24-3p was downregulated and RIPK1 was upregulated. Injection of miR-24-3p mimics and the RIPK1 adenovirus effectively led to increases in the expression levels of miR-24-3p and RIPK1, respectively. Fig. 5B proves that the upregulation of miR-24-3p led to the downregulation of RIPK1 by comparing the I/ $\mathrm{R}+\mathrm{miR}-24-3 p$ mimics group to the I/R group, which further verified the suppressive effect of miR-24-3p on RIPK1. Fig. 5C also shows the same changes in RIPK1 at the protein expression level. Fig. 5D, 5E and 5F show the expression levels of three enzymes that are closely related to ischemia/reperfusion injury. We determined that the activity of the enzymes was dramatically increased in the I/R group compared with that in the sham group $(P<0.01)$, but the addition of miR-24-3p (I/R + miR-24-3p group) decreased the enzyme expression levels back to the normal range $(P<0.01$, compared with those in the I/R group). In contrast, the addition of RIPK1 increased the activity of LDH, CK and CK-MB compared with the activity in the I/R group $(P<0.05)$. 


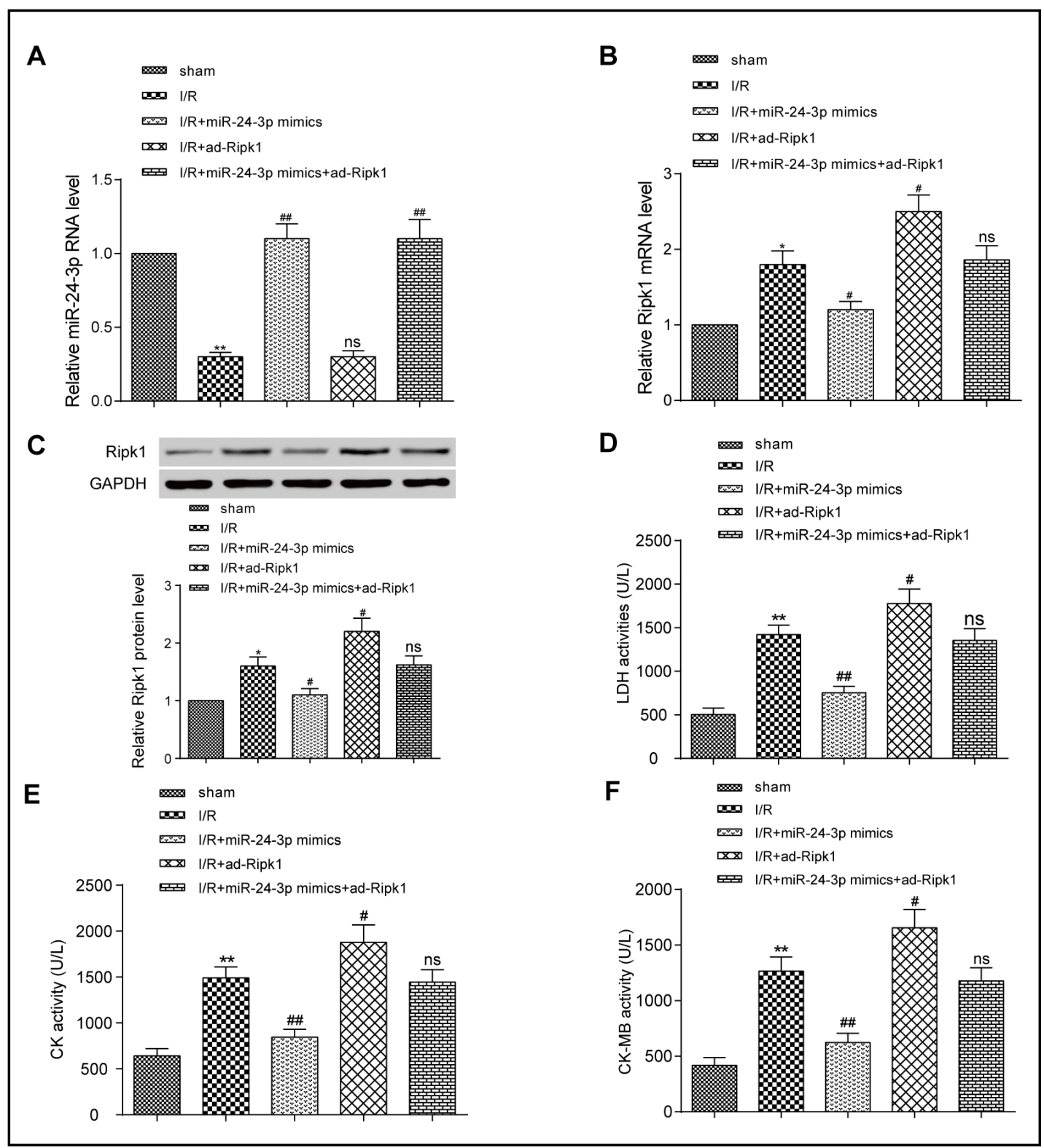

Fig. 5. Overexpression of Ripk1 and miR-24-3p influenced serum myocardial enzyme levels. (A) A qRTPCR assay measured the expression level of miR-24-3p in the five groups. (B) A qRT-PCR assay was used again to detect the mRNA expression of Ripk1 in the five groups. MiR-24-3p decreased the expression of Ripk1 in the I/R + miR-24-3p mimics group compared with that in the I/R group $(\mathrm{P}<0.05)$. (C) A Western blot assay was used to detect the protein expression of Ripk1 in the five groups. MiR-24-3p decreased the expression of Ripk1 in the I/R + miR-24-3p mimics group compared with that in the I/R group $(\mathrm{P}<0.05)$. (D) The concentration of LDH in the medium was measured in the five groups; the sham group was treated as a negative control group. (E) The concentration of CK in the medium was measured in the five groups. (F) The CK-MB concentration in the medium was also measured in the five groups. ( ${ }^{* *}$ means $\mathrm{P}<0.01$ compared with the $\mathrm{NC}$ group or the sham group; \#means $\mathrm{P}<0.05$, \#\#means $\mathrm{P}<0.01$ and ns means $\mathrm{P}>0.05$ compared with the I/R group). 


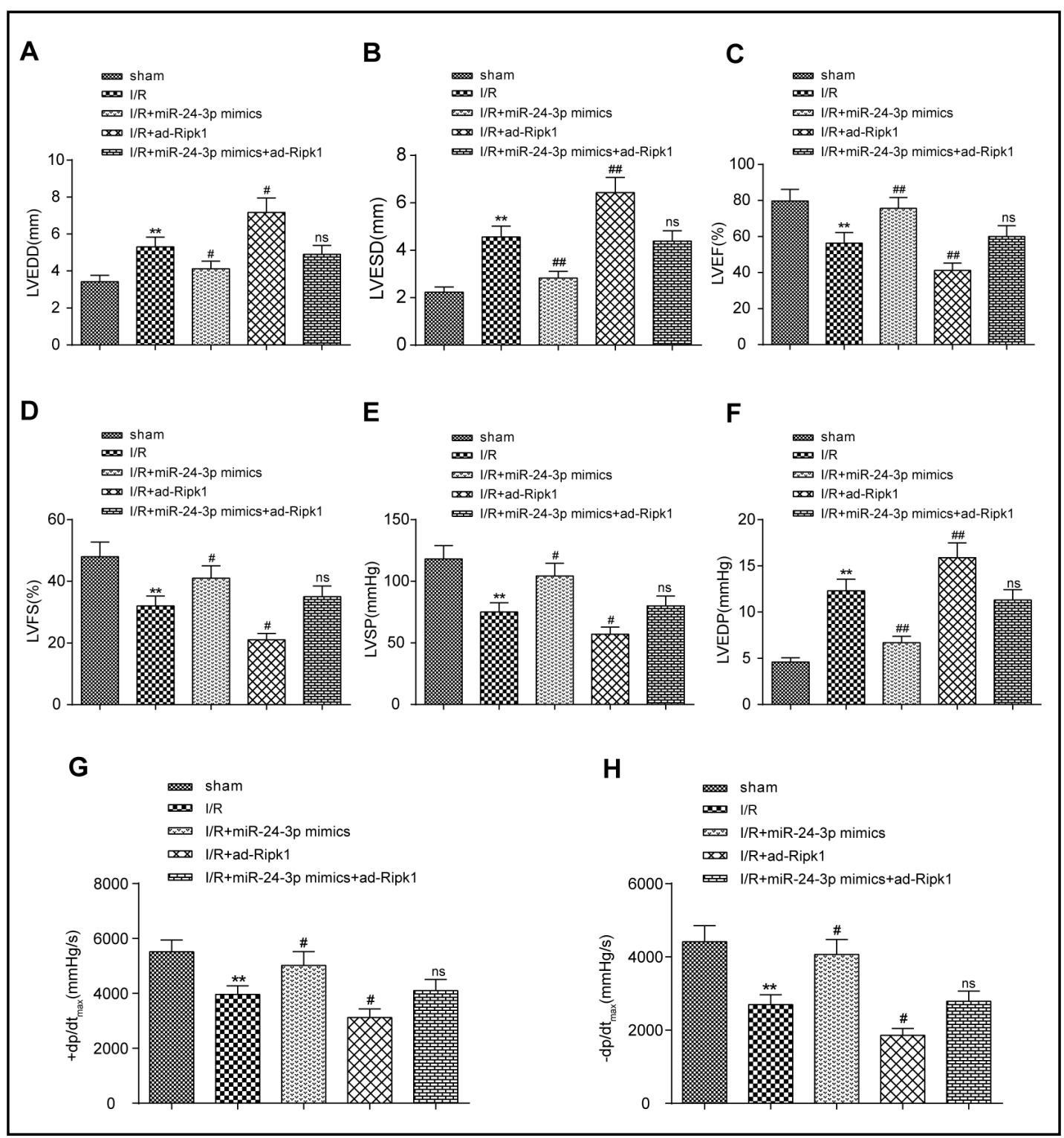

Fig. 6. The effects of Ripk1 and miR-24-3p on cardiac function. (A) LVEDD levels in the five transfected groups were measured. (B) LVESD levels in the five groups were measured, and the data for the I/R $+\mathrm{miR}$ 24-3p mimics group, the I/R + ad-Ripk1 group and the I/R + miR-24-3p mimics + ad-Ripk1 group were all compared with that of the I/R group to confirm the effects of miR-24-3p and Ripk1 on cardiac function in a mouse model. (C) LVEF levels. (D) LVFS levels. (E) The influences of miR-24-3p and Ripk1 on LVSP. (F) The influences of miR-24-3p and Ripk1 on LVEDP. (G) and $(\mathrm{H})$ The influences of miR-24-3p and Ripk1 on LV $\pm \mathrm{dp} / \mathrm{dt}_{\text {max }} \cdot{ }^{* *}$ means $\mathrm{P}<0.01$ compared with the sham group; ${ }^{\#}$ means $\mathrm{P}<0.05$, ${ }^{\# \#}$ means $\mathrm{P}<0.01$ and ns means $\mathrm{P}>0.05$ compared with the I/R group) (all analyses were performed after I/R surgery).

\section{The effects of RIPK1 and miR-24-3p on cardiac function}

After the ischemia/reperfusion experiment, the mice were examined by color Doppler echocardiography. Fig. 6A shows that the left ventricular end-diastolic dimension (LVEDD) was greatly enlarged in the I/R group from $3.5 \pm 0.1 \mathrm{~mm}$ (sham group) to approximately 5 $\mathrm{mm}(P<0.01)$. The addition of RIPK1 (I/R + ad-RIPK1 group) further increased the LVEDD to approximately $7 \mathrm{~mm}$ (compared with that of the I/R group, $P<0.05$ ). The I/R + miR-24$3 p$ mimics group had a significantly decreased LVEDD compared with that of the I/R group $(P<0.05)$, while the addition of RIPK1 (group I/R + miR-24-3p mimics + ad-RIPK1) restored 


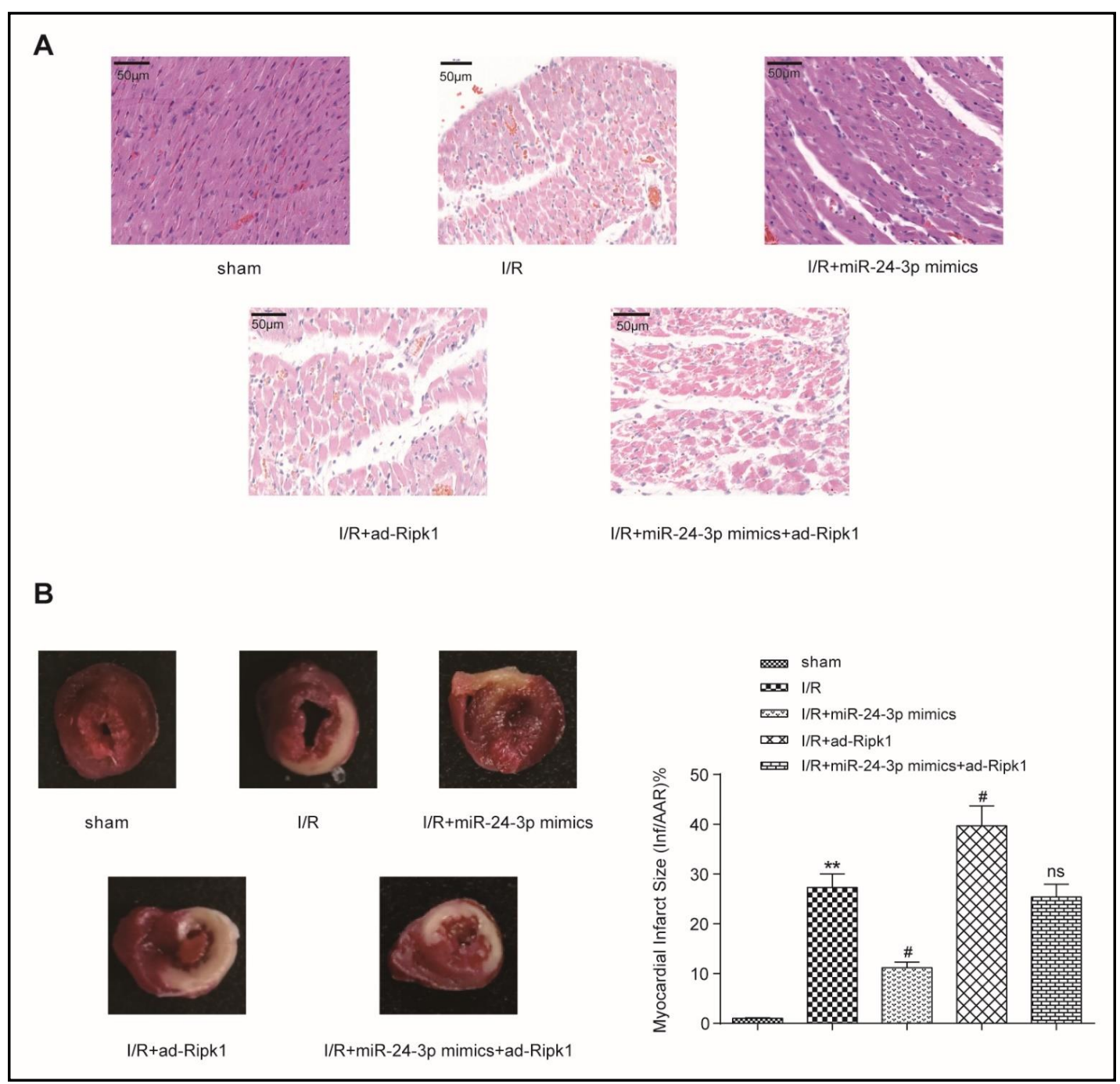

Fig. 7. The effects of Ripk1 and miR-24-3p on myocardial ischemia/reperfusion injury. (A) Hematoxylin and eosin staining of cardiomyocytes in the different transfection groups of mice. (B) TTC staining in the five different groups. The necrotic myocardium in the ischemic area is expressed in white, the ischemic myocardium is shown in red, and the nonischemic area is shown in blue. Image-Pro Plus was utilized to analyze the images and to calculate the ratio of the infarct area. ${ }^{* *}$ means $\mathrm{P}<0.01$ compared with the sham group; "means $\mathrm{P}<0.05$ and $n$ s indicates $\mathrm{P}>0.05$ compared with the I/R group) (all analyses were performed after I/R surgery).

the LVEDD to normal values. Fig. 6B shows the values of LVESD for the five different groups, which had the same tendency as the values of LVEDD. LVEF was obtained by dividing the stroke volume by the end-diastolic volume. This can measure the efficiency of the pumping in the systemic circulation (the normal range of LVEF is greater than 50\%). Fig. $6 \mathrm{C}$ shows that the I/R + ad-RIPK1 group had a lower LVEF than the I/R group $(P<0.01)$, and the addition of miR-24-3p (I/R + miR-24-3p mimics group) increased the value of LVEF $(P<0.01)$. Fig. $6 \mathrm{D}$ shows that the results for fraction shortening (FS) were similar to those for LVEF. Fig. $6 \mathrm{E}$ shows that the contraction capacity of the cardiac muscle as indicated by LVSP was decreased significantly in the I/R group $(P<0.01$, compared to that in the sham group) and was further decreased in the I/R + ad-RIPK1 group $(P<0.05$, compared to that in the $\mathrm{I} / \mathrm{R}$ group). The results of LVEDP detection are shown in Fig. $6 \mathrm{~F}$ and are totally opposite the results for LVSP. Fig. $6 \mathrm{G}$ and $6 \mathrm{H}$ show that the first derivative of the $\mathrm{LV}$ pressure $\left( \pm d p / d t_{\text {max }}\right)$ 


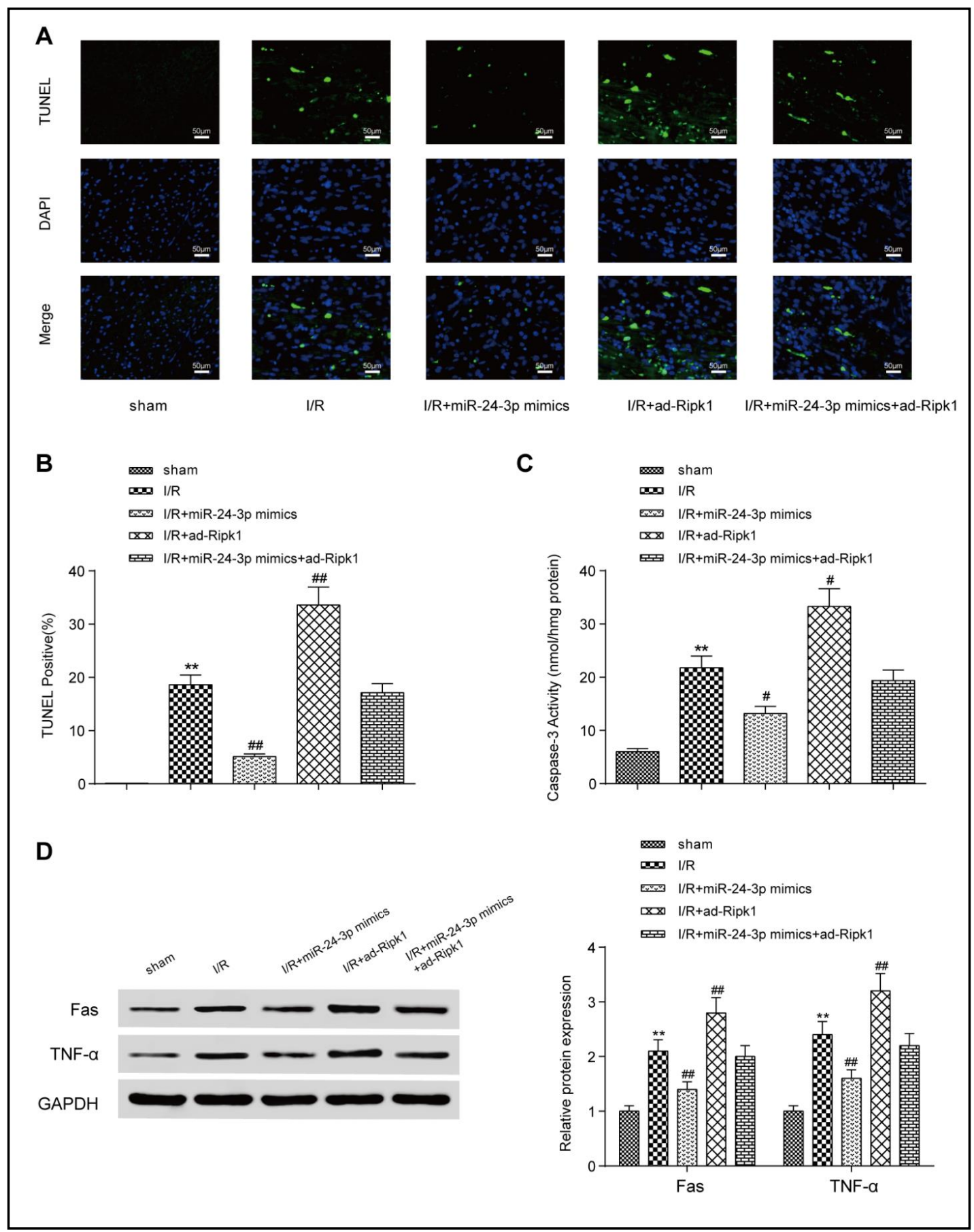

Fig. 8. The effects of Ripk1 and miR-24-3p on apoptosis and protein expression in the TNF pathway. (A) (B) TUNEL staining assay visualizing the apoptosis rates in the five transfected groups. Apoptotic nuclei are shown in green, and total nuclei are shown in blue. The Image-Pro Plus system was used for automatic counting. (C) Caspase-3 activity analysis in the five groups. (D) Western blot for relative proteins in the TNF pathway in each group. The expression levels of Fas and TNF- $\alpha$ in the heart in the I/R group were significantly increased compared with the levels in the heart in the sham group. MiR-24-3p mimics significantly decreased the expression levels of Fas and TNF- $\alpha$. Ripk1 significantly increased the expression levels of Fas and TNF- $\alpha$. ( ${ }^{* *}$ means $\mathrm{P}<0.01$ compared with the sham group, " means $\mathrm{P}<0.05$, ${ }^{\# \#}$ means $\mathrm{P}<0.01$ and ns means $\mathrm{P}>0.05$ compared with the I/R group). 


\section{Cellular Physiology Cell Physiol Biochem 2018;51:46-62

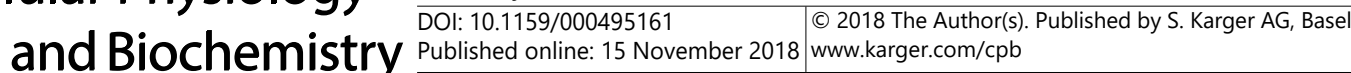 \\ Tan et al.: Role of miR-24-3p in Myocardial Ischemia/Reperfusion Injury}

in normal mice decreased significantly after I/R. $\pm \mathrm{dp} / \mathrm{dt} \mathrm{max}_{\text {max }}$ decreased significantly in the I/R + ad-RIPK1 group and increased in the I/R + miR-24-3p mimics group $(P<0.05$, compared to that in the I/R group).

The myocardial infarct size in the mouse model

Fig. 7A shows the hematoxylin and eosin staining of cardiomyocytes from the different transfection groups of the mice. In the sham group, normal cardiomyocytes were observed, and no hemorrhage or neutrophil granulocyte infiltration could be found. Myocardium injury was serious within the ischemic areas of the heart after I/R. Myocardial fibers were dissolved and infiltrated with inflammatory cells. Neutrophils accumulated within the ischemic areas of the heart after I/R. MiR-24-3p attenuated the myocardial injury induced by I/R. Hemorrhage, edema, and significant interstitial neutrophil granulocyte infiltration were observed in the I/R+ad-RIPK1 group. The area of myocardial infarction was measured after TTC staining. Fig. 7B illustrates that the infarct size of the myocardium occupied approximately $27 \%$ of the AAR, which was markedly larger than that of the sham group $(P<0.01)$. The infarct size in the AAR of the I/R + ad-RIPK1 group was dramatically increased compared with that of the I/R group $(P<0.05)$, while the infarction size of the mice in the $\mathrm{I} / \mathrm{R}+\mathrm{miR}-24-3 p$ mimic group was significantly decreased $(P<0.05)$. This result suggested that the addition of miR-24-3p could inhibit the effect of RIPK1 on mice and even reduce the infarction size.

The effects of RIPK1 and miR-24-3p on apoptosis and protein expression in the TNF pathway

TUNEL staining was used to illustrate that the apoptosis rate in the I/R group was significantly increased $(P<0.01$, compared to that in the sham group) (Fig. 8A and 8B). Furthermore, we found that miR-24-3p significantly decreased the apoptosis rate but that RIPK1 significantly increased the apoptosis rate compared with that in the I/R group $(P<0.01)$. According to the analysis of caspase-3 activity (Fig. $8 \mathrm{C})$, after I/R, the activity of caspase-3 increased and induced cardiac apoptosis in mice. In addition, compared with I/R alone, miR-24-3p significantly decreased the activity of caspase-3, and RIPK1 significantly increased the activity of caspase- 3 . Fas and TNF- $\alpha$ are proteins that are crucial in the TNF signaling pathway, and the detection of their expression levels in the five groups would strongly confirm the regulation of miR-24-3p and RIPK1 in the mouse model through the TNF signaling pathway. Fig. 8D shows that the expression levels of Fas and TNF- $\alpha$ in the heart in the I/R group increased significantly compared with those in the sham group. miR24-3p significantly decreased the expression levels of Fas and TNF- $\alpha$, but RIPK1 significantly promoted Fas and TNF- $\alpha$ expression compared to the expression in the I/R group $(P<0.01)$.

\section{Discussion}

The major findings of our study were based on experiments in a mouse model. The heatmap of the mRNA microarray showed that RIPK1 was highly expressed in the I/R injury mouse model, which led us to hypothesize that we could inhibit RIPK1 expression and reduce myocardial I/R injury. The research of Wang $\mathrm{K}$ et al. illustrated that RIPK1 was highly expressed in a myocardial ischemia/reperfusion injury mouse model, which is consistent with our results [13]. MicroRNA microarrays were re-analyzed to identify differentially expressed miRNAs. As a result, miR-24-3p was found to be expressed at low levels in the I/R mouse model, in contrast to the expression of RIPK1, and miR-24-3p was predicted to target RIPK1. Thus, miR-24-3p was chosen for further study. Aside from myocardial I/R injury, studies by Lorenzen JM have reported that miR-24 expression is also enriched in kidneys with I/R injury in mice $[11,15]$. Fiedler et al. demonstrated that the inhibition of endothelial miR-24 can decrease the myocardial infarct size in mice and result in preserved cardiac function [16]. Moreover, Qian et al. showed that the addition of miR-24 in the infarcted 


\section{Cellular Physiology Cell Physiol Biochem 2018;51:46-62

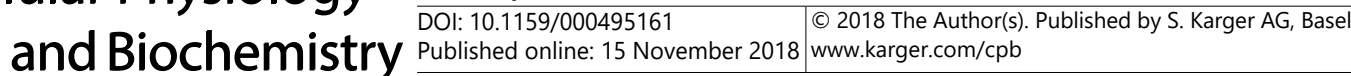 \\ Tan et al.: Role of miR-24-3p in Myocardial Ischemia/Reperfusion Injury}

hearts of mice suppressed cardiomyocyte apoptosis and reduced cardiac dysfunction [17]. All the studies described above support our major findings, and we hypothesize that there might be a targeting relationship between miR-24-3p and RIPK1 that regulates the ischemia/ reperfusion processes in mouse hearts.

Gene set enrichment analysis (GSEA) of GO and KEGG pathway analysis were applied. The GO analysis displayed the biological functions of the DEGs. Through the enrichment score analysis of KEGG, the TNF signaling pathway was found to be highly activated in myocardial I/R mice. The DEGs that were also included in the TNF signaling pathway were selected for further observation of their expression levels, and it is shown that all of these DEGs were highly upregulated in myocardial I/R mice. To confirm the microarray results, we conducted experiments to determine the expression levels of RIPK1 and miR-24-3p in $\mathrm{I} / \mathrm{R}$ mice compared to those in normal mice.

It was illustrated by a dual-luciferase reporter assay that miR-24-3p could directly bind to the 3'UTR of RIPK1 and regulate the expression of RIPK1. Subsequently, by detecting the activities of serum myocardial enzymes that are regarded as markers of ischemic myocardial damage, we found that the expression level of miR-24-3p in ischemia/reperfusion mice was negatively correlated with the levels of serum lactate dehydrogenase, creatine kinase and creatine kinase-mb form (LDH, CK and CK-MB) $[18,19]$. Patients with ischemic heart disease usually suffer from myocardial reperfusion after therapy, which might result in acute myocardial damage and adverse ventricular remodeling $[20,21]$. In addition, numerous studies have reported that myocardial I/R injury might induce alterations in cardiac hemodynamic parameters and further affect heart function [22]. In this study, our echocardiography results revealed that the levels of LVEF, LVFS, LVSP, and $\pm \mathrm{dp} / \mathrm{dt}_{\text {max }}$ in the I/R group were apparently lower than those in the sham group, while the levels of LVEDD, LVESD and LVEDP in the I/R group were markedly higher than those in the sham group. Nevertheless, the levels of LVEF, LVFS, LVSP, and $\pm d p / \mathrm{dt}_{\text {max }}$ in the I/R + miR-24-3p mimics group were significantly increased compared to those in the I/R group, while the levels of LVEDD, LVESD and LVEDP in the I/R + miR-24-3p group were decreased compared to those in the I/R group. Olof $\mathrm{G}$ et al. reported that circulating cardio-enriched miRNAs might be negatively correlated to the levels of LVEF [23]. Fan Q et al. revealed that the levels of LVFS and LVEF were decreased in a myocardial I/R injury mouse model compared with the levels in the sham group [24]. In conclusion, the results of the hemodynamic indexes proved that the contraction capacity of the cardiac muscle was impaired after myocardial I/R injury, and the addition of RIPK1 worsened the impairment. However, miR-24-3p improved the contraction capacity, indicating its cardioprotective effects. The results of all of the studies described above were consistent with the results of our study.

TTC staining showed that the infarct area of the I/R mice group was enlarged compared with that of the sham group and that the overexpression of miR-24-3p could decrease the area and restore it to the normal range. The results of the TUNEL assay in this study revealed that the apoptosis rate was markedly increased in mouse myocardial tissues subjected to ischemia/reperfusion, and the miR-24-3p mimics could markedly decrease cell apoptosis in myocardial tissue. The changes in the apoptosis rate in myocardial I/R mice were analyzed by caspase- 3 activity assays. The activity of the caspase- 3 enzyme was increased in the I/R group, while miR-24-3p significantly decreased the activity compared to that in the I/R group, which supported the results of the TUNEL assay. The expression of Fas and TNF- $\alpha$ was detected using Western blot since these proteins are crucial components in the TNF signaling pathway. The high expression of these proteins in the I/R + ad-RIPK1 group and the low expression in the I/R + miR-24-3p mimics group compared with that in the I/R group reflected RIPK1-mediated upregulation of the TNF pathway and miR-24-3p-mediated downregulation of the TNF pathway, which confirmed our previous demonstration. 


\section{Cellular Physiology Cell Physiol Biochem 2018;51:46-62 and Biochemistry \begin{tabular}{l|l} 
DOI: 10.1159/000495161 2018 The Author(s). Published by S. Karger AG, Basel \\
Publisher
\end{tabular}

\section{Conclusion}

In conclusion, our findings indicate that the suppression of RIPK1 might reduce the severity of ischemia/reperfusion injury and that miR-24-3p could serve as an inhibitor of RIPK1. The involvement of the TNF signaling pathway was confirmed in this study, and the direct association between miR-24-3p and RIPK1 was established using a luciferase assay. Our study shed new light on a potential therapy for I/R injury, and the inhibition of RIPK1 might be cardioprotective against I/R injury.

\section{Acknowledgements}

This work was supported by the National Natural Science Foundation of China (No. 81500313).

\section{Disclosure Statement}

The authors have no conflict of interest to declare.

\section{References}

1 Eltzschig HK, Eckle T: Ischemia and reperfusion--from mechanism to translation. Nat Med 2011;17:13911401.

-2 Yang J, Fan Z, Yang J, Ding J, Yang C, Chen L: microRNA-22 attenuates myocardial ischemia-reperfusion injury via an anti-inflammatory mechanism in rats. Exp Ther Med 2016;12:3249-3255.

-3 Yellon DM, Hausenloy DJ: Myocardial reperfusion injury. N Engl J Med 2007;357:1121-1135.

4 Qin D, Wang X, Li Y, Yang L, Wang R, Peng J, Essandoh K, Mu X, Peng T, Han Q Yu KJ, Fan GC: MicroRNA223-5p and -3p Cooperatively Suppress Necroptosis in Ischemic/Reperfused Hearts. J Biol Chem 2016;291:20247-20259.

5 Zhu H, Fan GC: Role of microRNAs in the reperfused myocardium towards post-infarct remodelling. Cardiovasc Res 2012;94:284-292.

6 Bijkerk R, de Bruin RG, van Solingen C, van Gils JM, Duijs JM, van der Veer EP, Rabelink TJ, Humphreys BD, van Zonneveld AJ: Silencing of microRNA-132 reduces renal fibrosis by selectively inhibiting myofibroblast proliferation. Kidney Int 2016;89:1268-1280.

7 Zuo Y, Wang Y, Hu H, Cui W: Atorvastatin Protects Myocardium Against Ischemia-Reperfusion Injury Through Inhibiting miR-199a-5p. Cell Physiol Biochem 2016;39:1021-1030.

8 Wang X, Zhang X, Ren XP, Chen J, Liu H, Yang J, Medvedovic M, Hu Z, Fan GC: MicroRNA-494 targeting both proapoptotic and antiapoptotic proteins protects against ischemia/reperfusion-induced cardiac injury. Circulation 2010;122:1308-1318.

-9 Zhu WS, Guo W, Zhu JN, Tang CM, Fu YH, Lin QX, Tan N, Shan ZX: Hsp90aa1: a novel target gene of miR-1 in cardiac ischemia/reperfusion injury. Sci Rep 2016;6:24498.

10 Zhu J, Yao K, Wang Q, Guo J, Shi H, Ma L, Liu H, Gao W, Zou Y, Ge J: Ischemic Postconditioning-Regulated miR-499 Protects the Rat Heart Against Ischemia/Reperfusion Injury by Inhibiting Apoptosis through PDCD4. Cell Physiol Biochem 2016;39:2364-2380.

-11 Lorenzen JM, Kaucsar T, Schauerte C, Schmitt R, Rong S, Hubner A, Scherf K, Fiedler J, Martino F, Kumarswamy R, Kolling M, Sorensen I, Hinz H, Heineke J, van Rooij E, Haller H, Thum T: MicroRNA-24 antagonism prevents renal ischemia reperfusion injury. J Am Soc Nephrol 2014;25:2717-2729.

12 Christofferson DE, Li Y, Yuan J: Control of life-or-death decisions by RIP1 kinase. Annu Rev Physiol 2014;76:129-150.

13 Wang K, Liu F, Liu CY, An T, Zhang J, Zhou LY, Wang M, Dong YH, Li N, Gao JN, Zhao YF, Li PF: The long noncoding RNA NRF regulates programmed necrosis and myocardial injury during ischemia and reperfusion by targeting miR-873. Cell Death Differ 2016;23:1394-1405. 


\section{Cellular Physiology Cell Physiol Biochem 2018;51:46-62

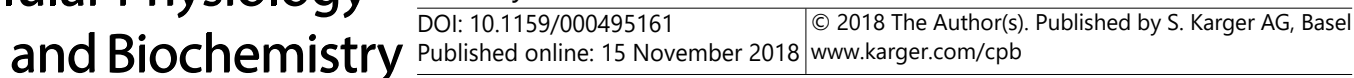 \\ Tan et al.: Role of miR-24-3p in Myocardial Ischemia/Reperfusion Injury}

14 Oerlemans MI, Liu J, Arslan F, den Ouden K, van Middelaar BJ, Doevendans PA, Sluijter JP: Inhibition of RIP1-dependent necrosis prevents adverse cardiac remodeling after myocardial ischemia-reperfusion in vivo. Basic Res Cardiol 2012;107:270.

15 Lorenzen JM: Vascular and circulating microRNAs in renal ischaemia-reperfusion injury. J Physiol 2015;593:1777-1784.

-16 Fiedler J, Jazbutyte V, Kirchmaier BC, Gupta SK, Lorenzen J, Hartmann D, Galuppo P, Kneitz S, Pena JT, SohnLee C, Loyer X, Soutschek J, Brand T, Tuschl T, Heineke J, Martin U, Schulte-Merker S, Ertl G, Engelhardt S, Bauersachs J, Thum T: MicroRNA-24 regulates vascularity after myocardial infarction. Circulation 2011;124:720-730.

-17 Qian L, Van Laake LW, Huang Y, Liu S, Wendland MF, Srivastava D: miR-24 inhibits apoptosis and represses Bim in mouse cardiomyocytes. J Exp Med 2011;208:549-560.

18 Ke ZP, Xu P, Shi Y, Gao AM: MicroRNA-93 inhibits ischemia-reperfusion induced cardiomyocyte apoptosis by targeting PTEN. Oncotarget 2016;7:28796-28805.

19 Pan Z, Sun X, Ren J, Li X, Gao X, Lu C, Zhang Y, Sun H, Wang Y, Wang H, Wang J, Xie L, Lu Y, Yang B: miR-1 exacerbates cardiac ischemia-reperfusion injury in mouse models. PLoS One 2012; 7:e50515.

20 Song CL, Liu B, Diao HY, Shi YF, Li YX, Zhang JC, Lu Y, Wang G, Liu J, Yu YP, Guo ZY, Wang JP, Zhao Z, Liu JG, Liu YH, Liu ZX, Cai D, Li Q: The protective effect of microRNA-320 on left ventricular remodeling after myocardial ischemia-reperfusion injury in the rat model. Int J Mol Sci 2014;15:17442-17456.

21 Hausenloy DJ, Yellon DM: Myocardial ischemia-reperfusion injury: a neglected therapeutic target. J Clin Invest 2013;123:92-100.

22 Toldo S, Seropian IM, Mezzaroma E, Van Tassell BW, Salloum FN, Lewis EC, Voelkel N, Dinarello CA, Abbate A: Alpha-1 antitrypsin inhibits caspase- 1 and protects from acute myocardial ischemia-reperfusion injury. J Mol Cell Cardiol 2011;51:244-251.

$>23$ Gidlof O, Smith JG, Miyazu K, Gilje P, Spencer A, Blomquist S, Erlinge D: Circulating cardio-enriched microRNAs are associated with long-term prognosis following myocardial infarction. BMC Cardiovasc Disord 2013;13:12.

-24 Fan Q, Chen M, Zuo L, Shang X, Huang MZ, Ciccarelli M, Raake P, Brinks H, Chuprun KJ, Dorn GW, 2nd, Koch WJ, Gao E: Myocardial Ablation of G Protein-Coupled Receptor Kinase 2 (GRK2) Decreases Ischemia/ Reperfusion Injury through an Anti-Intrinsic Apoptotic Pathway. PLoS One 2013;8:e66234. 